\title{
DERIVATIONS OF NILPOTENT LIE ALGEBRAS
}

J. DIXMIER AND W. G. LISTER

In a recent note Jacobson proved [1] that, over a field of characteristic 0 , a Lie algebra with a nonsingular derivation is nilpotent. $\mathrm{He}$ also noted that the validity of the converse was an open question. The purpose of this note is to supply a strongly negative answer to that question and to point out some of the immediate problems which this answer raises.

Suppose then that $\Phi$ is a field of characteristic 0 and that $\mathfrak{R}$ is the 8 dimensional algebra over $\Phi$ described in terms of a basis $e_{1}, e_{2}, \cdots$, $e_{8}$ by the following multiplication table:

(1) $\left[e_{1}, e_{2}\right]=e_{5}$

(2) $\left[e_{1}, e_{3}\right]=e_{6}$,

(3) $\left[e_{1}, e_{4}\right]=e_{7}$,

(4) $\left[e_{1}, e_{5}\right]=-e_{8}$,

(5) $\left[e_{2}, e_{3}\right]=e_{8}$,
(6) $\left[e_{2}, e_{4}\right]=e_{6}$,

(7) $\left[e_{2}, e_{6}\right]=-e_{7}$,

(8) $\left[e_{3}, e_{4}\right]=-e_{5}$,

(9) $\left[e_{3}, e_{5}\right]=-e_{7}$,

(10) $\left[e_{4}, e_{6}\right]=-e_{8}$.

In addition $\left[e_{i}, e_{j}\right]=-\left[e_{j}, e_{i}\right]$ and for $i<j\left[e_{i}, e_{j}\right]=0$ if it is not in the table above. Note that all triple products $\left[\left[e_{i} e_{j}\right] e_{k}\right]$ vanish if one index is $>4$. It is convenient to use a symmetry in the table above. Denote by $A$ the linear transformation induced in $\mathfrak{R}$ by the mapping

$$
\left(\begin{array}{rrrrrrrr}
e_{1} & e_{2} & e_{3} & e_{4} & e_{5} & e_{6} & e_{7} & e_{8} \\
e_{3} & e_{4} & e_{1} & e_{2} & -e_{5} & -e_{6} & -e_{8} & -e_{7}
\end{array}\right) .
$$

A direct check shows that $A$ is an automorphism of $\mathfrak{R}$. By observing

$$
\left[\left[\begin{array}{ll}
e_{1} & e_{2}
\end{array}\right] e_{3}\right]+\left[\left[\begin{array}{ll}
e_{2} & e_{3}
\end{array}\right] e_{1}\right]+\left[\left[e_{3} e_{1}\right] e_{2}\right]=e_{7}-e_{7}=0
$$

and

$$
\left[\left[\begin{array}{ll}
e_{1} & e_{2}
\end{array}\right] e_{4}\right]+\left[\left[\begin{array}{ll}
e_{2} & e_{4}
\end{array}\right] e_{1}\right]+\left[\left[\begin{array}{ll}
e_{4} & e_{1}
\end{array}\right] e_{2}\right]=0,
$$

and by applying $A$ to each we conclude that $\ell$ is a Lie algebra.

Since $\mathfrak{R}^{2}=\left\{e_{5}, e_{6}, e_{7}, e_{8}\right\}, \mathfrak{R}^{3}=\left\{e_{7}, e_{8}\right\}, \mathfrak{R}^{4}=\{0\}, \mathfrak{\&}$ is nilpotent.

ThEOREM. If $D$ is a derivation of $\mathbb{R}$ then $\mathfrak{R} D \subset \mathbb{R}^{2}$; hence every derivation is nilpotent.

Proof. Suppose $e_{i} D=\sum \delta_{i j} e_{j}, 1 \leqq i \leqq 8,1 \leqq j \leqq 8$. The equations

Received by the editors March 1, 1956. 


$$
\begin{aligned}
{\left[\begin{array}{ll}
e_{1} e_{2}
\end{array}\right] D=e_{5} D } & =\delta_{55} e_{5}+\delta_{56} e_{6}+\delta_{57} e_{7}+\delta_{58} e_{8}, \\
{\left[e_{1} D, e_{2}\right.} & =\delta_{11} e_{5}-\delta_{14} e_{6}+\delta_{16} e_{7}-\delta_{13} e_{8}, \\
{\left[e_{1}, e_{2} D\right] } & =\delta_{22} e_{5}+\delta_{23} e_{6}+\delta_{24} e_{7}-\delta_{25} e_{8}
\end{aligned}
$$

imply

$\delta_{55}=\delta_{11}+\delta_{22}, \quad \delta_{56}=\delta_{23}-\delta_{14}, \quad \delta_{57}=\delta_{16}+\delta_{24}, \quad \delta_{58}=-\delta_{13}-\delta_{25}$.

With the observation that for $i \leqq 4$ and $5 \leqq k \leqq 8$ there is exactly one $j \leqq 6$ for which $\left[e_{i}, e_{j}\right]= \pm e_{k}$ it follows from (2) that

$$
\delta_{65}=\delta_{14}+\delta_{32}, \quad \delta_{66}=\delta_{11}+\delta_{33}, \quad \delta_{67}=\delta_{15}+\delta_{34}, \quad \delta_{68}=\delta_{12}-\delta_{35},
$$

from (3) that

$$
\delta_{77}=\delta_{11}+\delta_{44}, \quad \delta_{78}=\delta_{16}-\delta_{45}, \quad \delta_{13}=\delta_{42}, \quad \delta_{12}=-\delta_{43}
$$

and from (4) that

$$
\delta_{87}=\delta_{13}, \quad \delta_{88}=\delta_{11}+\delta_{55} .
$$

The automorphism $A$ transforms $D$ into a derivation $D^{*}$ by $A^{-1} D A$ $=D^{*}$ and this implies that with each equation $\delta_{i j}=\delta_{k l}+\delta_{m n}$ there is also valid $\delta_{\alpha(i), \alpha(j)}=\delta_{\alpha(k), \alpha(l)}+\delta_{\alpha(m), \alpha(n)}$ where $\alpha$ is the permutation of $\{-8,-7, \cdots, 7,8\}$ induced by $A$ and where $\delta_{(-1) p_{i},(-1) q_{j}}$ $=(-1)^{p+q} \delta_{i j}$. $D$ operating on (6) provides

$\delta_{65}=-\delta_{23}-\delta_{41}, \quad \delta_{66}=\delta_{22}+\delta_{44}, \quad \delta_{67}=\delta_{21}-\delta_{46}, \quad \delta_{68}=\delta_{26}+\delta_{43}$, and (7) gives

$$
\delta_{77}=\delta_{66}+\delta_{22}, \quad \delta_{78}=\delta_{24} .
$$

From the vanishing of $\left[e_{1}, e_{6}\right]$ follows $\delta_{12}=0, \delta_{14}=-\delta_{65}$ and from $\left[e_{2}, e_{5}\right]=0, \delta_{21}=0, \delta_{23}=-\delta_{56}$. Again, another set of equations is obtained by applying $A$.

Among the ten relations of the form $\delta_{i i}+\delta_{j j}=\delta_{k k}$, eight are linearly independent so that $\delta_{i i}=0$ for $i=1,2, \cdots, 8$. The relations

$$
\delta_{78}=\delta_{31}=\delta_{24}=\delta_{16}-\delta_{45}
$$

and

$$
\delta_{57}=\delta_{45}-\delta_{31}=\delta_{16}+\delta_{24}
$$

imply

$$
\delta_{57}=\delta_{16}=\delta_{45} \text { and } \quad \delta_{24}=\delta_{78}=\delta_{31}=0,
$$

and there are also 


$$
\delta_{58}=-\delta_{36}=-\delta_{25}, \quad \delta_{42}=\delta_{87}=\delta_{13}=0 .
$$

$\delta_{21}=0$ implies $\delta_{43}=0$. The relations $\delta_{68}=\delta_{12}-\delta_{35}=\delta_{26}+\delta_{43}$ imply $\delta_{68}=-\delta_{35}=\delta_{26}$, and thus also $\delta_{67}=\delta_{15}=-\delta_{46}$. Since $\delta_{23}=-\delta_{56}$ and $-\delta_{65}$ $=\delta_{23}+\delta_{41}=-\delta_{14}-\delta_{32}=\delta_{41}=\delta_{14}$,

$$
\delta_{65}=\delta_{41}=\delta_{23}=\delta_{14}=\delta_{32}=\delta_{56}=0 .
$$

The matrix of $D$ is therefore:

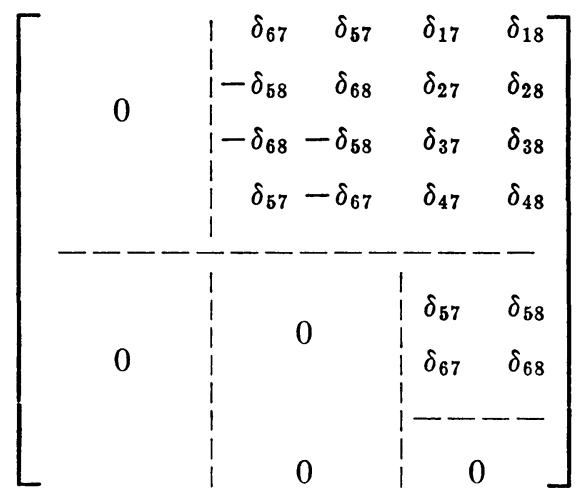

The derivation algebra $\mathfrak{D}$ is 12 dimensional and the algebra $\Im$ of inner derivations 6 dimensional. Every linear transformation sending $\mathfrak{R}$ into the center of $\mathfrak{R}$ and $\mathfrak{R}^{2}$ into 0 is a derivation. The ideal $\mathfrak{D}_{0}$ of these derivations is 8 dimensional and intersects $\Im$ in a 2 dimensional space. In a sense then $\mathfrak{R}$ has as few outer derivations as possible. More precisely,

$$
\mathfrak{N}=\mathfrak{D}_{0}+\mathfrak{\Im} .
$$

The existence of $\&$ suggests the consideration of a subclass of nilpotent algebras which might prove more tractable than the entire class. To this end, for any algebra $\mathfrak{N}$ with derivation algebra $\mathfrak{D}$, let

$$
\mathfrak{R}^{[1]}=\mathfrak{N D}=\left\{\sum x_{i} D_{i} \mid x_{i} \in \mathfrak{N}, D_{i} \in \mathfrak{D}\right\},
$$

and let

$$
\mathfrak{N}^{[k+1]}=\mathfrak{N}^{[k]} \mathfrak{D} .
$$

$\mathfrak{N}$ could be called characteristically nilpotent if for some $k, \mathfrak{N}^{[k]}=0$. The algebra $\mathfrak{R}$ is characteristically nilpotent and for any such algebra $\mathfrak{R}$,

(1) if $\mathfrak{R}$ is an ideal of a solvable algebra $\Re$ then either $\Re^{k} \subset \mathfrak{N}$ for any $k$ or $\Re$ is nilpotent, 
(2) if $\mathfrak{M}$ is an algebra with nil-radical $\mathfrak{N}$ then $\mathfrak{M}=\mathfrak{R} \oplus \mathfrak{S}$ for some semi-simple ideal 5 .

One might ask whether there is an intrinsic characterization of such algebras, and a general method for constructing them all.

The algebra $\&$ has an additional property which may be shared by all characteristically nilpotent algebras: $\&$ is not the derived algebra of any Lie algebra. To see this, observe that $\mathfrak{R}^{[1]}=\mathfrak{R D}=\mathfrak{R}^{2}$ so that if $\mathfrak{R} \subset \mathfrak{M}$ and $\mathfrak{M}^{2}=\mathfrak{R}$, then $[\mathfrak{R M}] \subset \mathfrak{R}^{[1]}=\mathfrak{R}^{2}$. This implies $\mathfrak{R}^{2}=[\mathfrak{R}[\mathfrak{M M}]]$ $\subset[[\mathbb{R M}] \mathfrak{M}] \subset\left[\mathfrak{R}^{2} \mathfrak{M}\right] \subset\left[\mathfrak{R}^{2} \mathfrak{R}\right]$, and this contradicts the nilpotency of ?.

\section{REFERENCE}

1. N. Jacobson, $A$ note on automorphisms and derivations of Lie algebras, Proc. Amer. Math. Soc. vol. 6 (1955) pp. 281-283.

University OF PARIS AND

BROWN UNIVERSITY 